A rare case of disseminated peritoneal cylindromatosis was presented. A young woman was admitted to hospital after removal of a right ovarian cystic tumour. Multiple histological reexaminations suggested the presence of a dermoid cyst, i.e. adult teratoma additionally with granulosa cell tumour and endometrioid cancer. Six years after complete removal of neoplastic foci, a routine examination revealed multiple nodular lesions inside the pelvis and in the pelvic peritoneum. Multiple histological studies confirmed the presence of a cylindroma. Based on the broad histochemistry and immunohistochemistry (periodic acid Shiff, Ber-EP4, p63, CKAE1/AE3, CEA positive, as opposed to negative results with inhibin, calretinin, CK7 and only focally positive for EMA; Ki 67 mitotic index did not exceed $5 \%$ of cells), granulosa cell tumour and endometrioid cancer was excluded. A comparison of the surgical material with the specimens taken from the primary lesion operated eight years earlier showed a similarity of histological images. Our case of peritoneal dissemination of eccrine cylindroma arising from dermoid cyst suggests the introduction of the term peritoneal cylindromatosis for similar cases.

Key words: adult teratoma, cylindroma, peritoneum, tumour dissemination.

Contemp Oncol (Pozn) 2014; 18 special issue DOI: $10.5114 /$ wo.2014.40605

\section{Peritoneal cylindromatosis - a rare teratomatous dissemination}

\author{
Ewa Zembala-Nożyńska', Dariusz Lange ${ }^{1}$, Ewa Stobiecka', Mariusz Kryj², \\ Marcin Zeman ${ }^{2}$, Maciej Wideł², Łukasz Zarudzki ${ }^{3}$
}

${ }^{1}$ Tumour Pathology Department, Maria Skłodowska-Curie Memorial Cancer Centre and Institute of Oncology Gliwice Branch, Poland

${ }^{2}$ Oncology Surgery Clinic, Maria Skłodowska-Curie Memorial Cancer Centre and Institute of Oncology Gliwice Branch, Poland

${ }^{3}$ Radiodiagnostics Department, Maria Skłodowska-Curie Memorial Cancer Centre and Institute of Oncology Gliwice Branch, Poland

\section{Introduction}

Ovarian teratomas are a particular group of tumours arising from primitive germ cells (30\% of primary ovarian tumours). Depending on their lineage, they can be divided into two-phasic or three-phasic tumours. The WHO classification distinguishes mature (27-44\% of germ cell tumours) and immature teratomas (3\%), cystic teratomas and dermoid cysts (over 50\%) [1]. Of these developmental lesions, mature teratomas are the most frequent. They constitute a large group and are the predominant type among benign ovarian tumours.

Teratomas most commonly present in the third decade of life, but have been reported at any age between 2 to 80 years old. They can have the form of a cystic or a solid tumour composed of a variety of mature tissues. Usually they are detected accidentally, rarely giving clinical signs related to the size of the tumour or complications, such as torsion, with the associated pain, haemorrhage or, very rarely, spontaneous rupture.

The most histogenetically surprising group are mature teratomas with a benign or malignant tissue component, especially those combining benign pattern with intraperitoneal dissemination or dissemination to distant organ sites, which makes the tumour clinically malignant. We describe the case of a young woman with peritoneal dissemination of a cylindroma component.

\section{Case report}

A 35-year-old woman was admitted to the Oncology Institute (Gliwice, Poland) after the removal of a right ovarian cystic tumour performed laparoscopically 8 years earlier. Family history was negative for the presence of malignancies or other tumours. The primary clinical diagnosis was preceded by increasing abdominal pains and irregular menstrual cycles. The ultrasound revealed single cystic tumour localized in the right ovary. Additional laboratory findings showed no significant differences. Earlier histological examination of the surgical specimen revealed the presence of a cystic tumour with elements of a dermoid cyst, i.e. adult teratoma and, additionally, granulosa cell tumour (Fig. 1). Re-examination of the histological preparations with immunohistochemistry, performed at the Oncology Institute, suggested well-differentiated endometrial ovarian cancer (CK 18-positive, CK8-negative, inhibin-negative, chromogranin-negative, mucicarmine-positive). Moreover, the patient reported pregnancy of four weeks. In view of the diagnosis, a more extensive surgical resection, encompassing early abortion, was suggested. The pregnancy was removed together with the remaining remnants of right uterine appendages, the omentum and the 


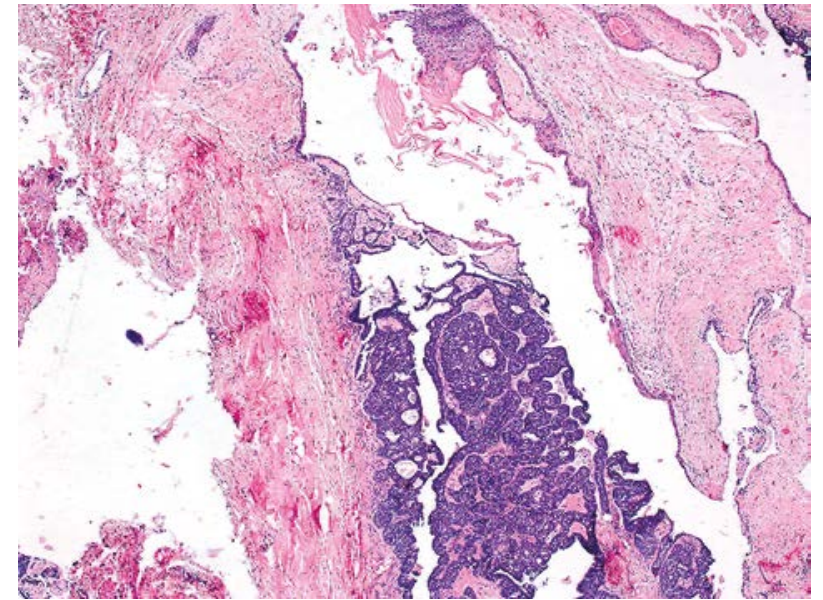

Fig. 1. Histopathology of primary ovarian tumour. Component of dermoid cyst and misdiagnosed granulosa cell tumour. HE, magnification $40 \times$

vermiform appendix. The histological examination did not confirm neoplastic foci. Regular follow-up visits showed no progression of the disease and 6 years after the surgical treatment, the patient gave birth to a healthy child, free of complications. A year and a half later, a routine examination revealed bilateral nodular lesions of approx. $20 \mathrm{~mm}$ in diameter in the right iliac wing appendages. In the site of the right appendages, an ultrasound examination showed 4 solid tumours: the largest was $20 \mathrm{~mm} \times 15 \mathrm{~mm} \times 18 \mathrm{~mm}$ with visible vascularization, whilst the three smaller tumours were $12 \mathrm{~mm} \times 7 \mathrm{~mm}, 16 \mathrm{~mm} \times 9 \mathrm{~mm}$ and $12 \mathrm{~mm}$ $\times 7 \mathrm{~mm}$. In the site of the left appendages, the ovary of $35 \mathrm{~mm} \times 23 \mathrm{~mm}$ was observed together with 5 solid tumours ranging from $15 \mathrm{~mm} \times 11 \mathrm{~mm}$ to $17 \mathrm{~mm} \times 14 \mathrm{~mm}$. Computed tomography revealed numerous interconnected polycyclic nodular structures in the vesicouterine pouch and the rectouterine pouch, as well as in the left ovarian fossa (Fig. 2). The largest lesion, at $25 \mathrm{~mm} \times 48 \mathrm{~mm} \times 43 \mathrm{~mm}$, was located between the urinary bladder, the body of the uterus and the rectum. The lesions showed clear heterogeneous contrast enhancement. Similar oval-shaped structures of dimensions ranging from $16 \mathrm{~mm} \times 11 \mathrm{~mm}$ to $17 \mathrm{~mm} \times 14 \mathrm{~mm}$ stretched in a stringy manner, medial to the external iliac vessels, along the left round ligament of the uterus for approx. $8 \mathrm{~cm}$, reaching the rectus abdominis muscle on the left side. In the proximity of the subcutaneous fatty tissue, a similar tumour of approx. $10 \mathrm{~mm}$ in diameter was observed. Another similar lesion of $24 \mathrm{~mm}$ $\times 25 \mathrm{~mm}$ was found in the area corresponding to the right appendages. The presence of further tumours of 4-14 mm was observed within the pelvic peritoneum, by the external contours of the sigmoid colon loop wall and the transverse colon. All of the above radiological manifestations suggested a peritoneal dissemination of neoplastic lesions arising from the left appendages. However, the laboratory results were within normal limits (LDH - $123.3 \mathrm{IU} / \mathrm{l}$; AFP - $1.43 \mathrm{IU} / \mathrm{ml}$; Ca 125 - $12.73 \mathrm{U} / \mathrm{ml}$; CEA - 1.05 ng/ml). Laparotomy was repeated with a view to perform hysterectomy and remove the remaining left appendages, the parietal peritoneum of the pelvis minor, the retroperitoneal lymph

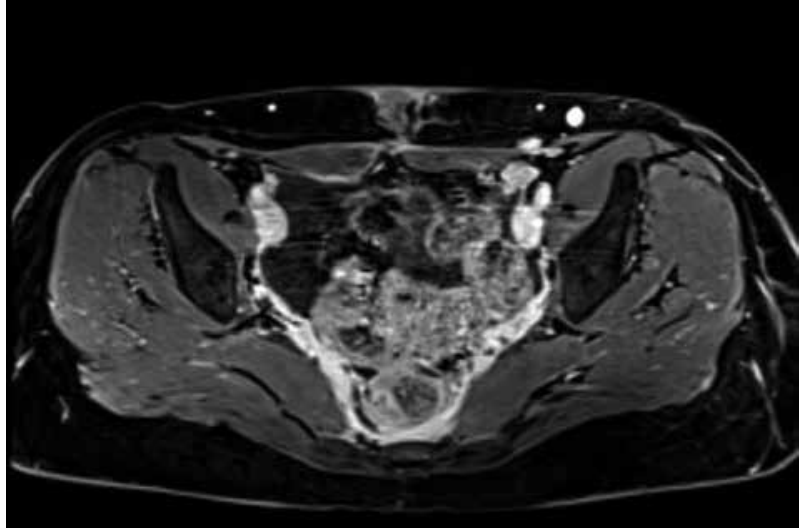

Fig. 2. CT-scan reveals peritoneal disseminated tumours

nodes, the omentum and the pelvic tumours. Intraoperatively, numerous tumours of the parietal peritoneum of 5 to $30 \mathrm{~mm}$ diameter were found in the pelvis minor. The uterus, left appendages and the mesenteric lymph nodes were removed, together with numerous pelvis minor tumours clustered into conglomerates of up to $85 \mathrm{~mm}$ in diameter. The intraoperative histological examination revealed a neoplastic infiltration, referred for further diagnosis with paraffin and immunohistochemical tests. The uterus, left appendages and the lymph nodes showed no neoplastic changes. A comparison of the surgical material with the specimens taken from the primary lesion operated eight years earlier showed a similarity of histological images. More comprehensive histological diagnostics, including histochemical and immunohistochemical examinations, confirmed the presence of a well-differentiated neoplasm of epithelial origin and malignant clinical course. However, the characteristic PAS-positive reaction in basement membranes was typical of cylindroma tumours arising from skin appendages. Immunohistochemical stainings, including Ber-EP4, p63, CKAE1/AE3 and CEA, showed a positive reaction. Based on the negative results of inhibin and calretinin, granulosa cell tumour was excluded. Endometrioid cancer was excluded based on negative reactions for CK7 and only focally positive for EMA. The Ki 67 mitotic index did not exceed $5 \%$ of cells in the field of view. Based on the full clinical course and morphological manifestations, malignant teratoma arising from the epithelial component of skin appendages with eccrine-apocrine differentiation in the form of a cylindroma, was diagnosed (Figs. 3-6). The patient was evaluated periodically (last control in January 2013) as free of recidiva in CT-scans.

\section{Discussion}

The puzzling peritoneal dissemination of mature teratoma may have various forms. Cases of implantation of mucous cells into the peritoneum, resulting in the development of peritoneal pseudomyxoma, are commonly known [2]. Alexander et al. [3] reported a case of peritoneal gliomatosis resulting from the implantation of a mature neuroglia during the recurrence of mature teratoma treated surgically. Targnion et al. [4] described the same phenomenon with the nosologically unclear term cancerous dissemination. Sibio et al. [5] described peritoneal 


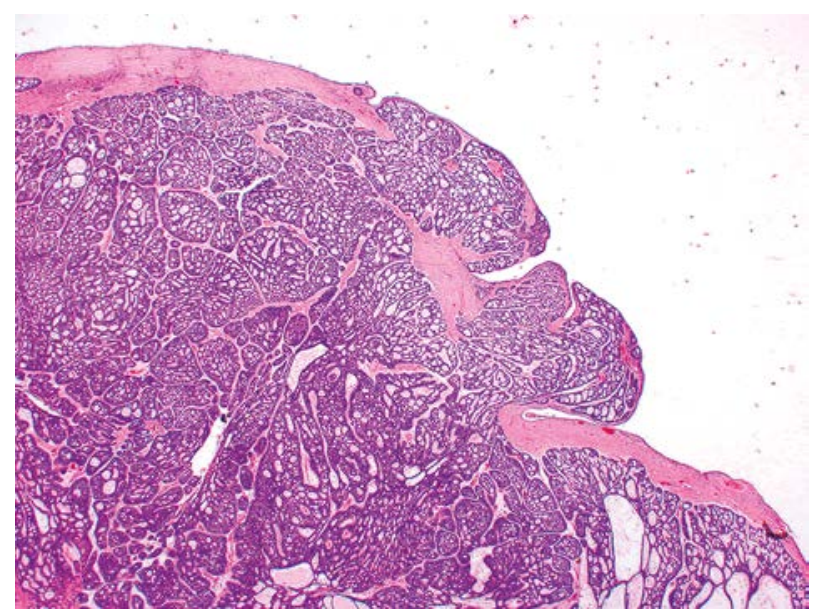

Fig. 3. Histopathology of recurrent peritoneal tumour with typical cylindromatous component. HE, magnification 100x

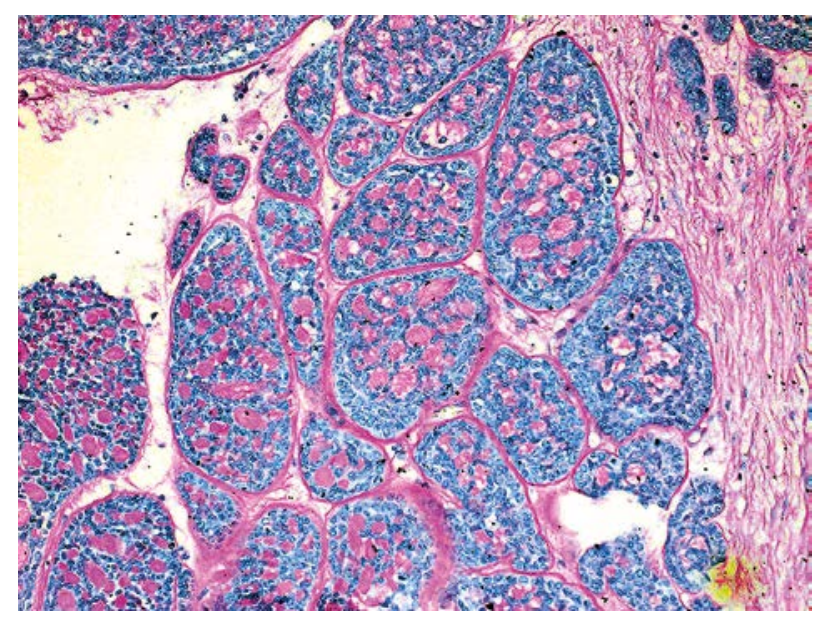

Fig. 5. Periodic acid Shiff stain reveals positively stained tumour spaces and basement membranes. Magnification 200x

strumosis as dissemination of one of the components of a dermoid cyst. Analogically, our case of peritoneal dissemination of eccrine cylindroma seems to warrant the introduction of the term peritoneal cylindromatosis. Of all teratomas connected with the dermoid cyst and encompassing a huge variety of lesions, from clinically benign to malignant, originating from all germ layers, there is only a small group of tumours arising from skin appendages, such as malignant melanoma, melanocytic naevus, sebaceous adenoma, sebaceous carcinoma and teratoma with a flat tumour component (teratoma with malignant transformation) $[6,7]$. We found no publications regarding the cylindroma component in teratomas. Eccrine cylindroma belongs to a huge group of tumours arising from skin appendages with eccrine secretion. It occurs most frequently on the head skin, in the form of a single tumour or, in rare cases of Ancell-Spiegler syndrome, in the form of numerous tumours covering most of the skin of the head, sometimes the face and the upper half of the body (autosomal dominant, linked to the CYLD1 gene on chromosome $16 \mathrm{q}$ 12-13) [8].

Cylindroma includes two cell populations: compact outer basal cells and polygonal cells with a more amphophilic

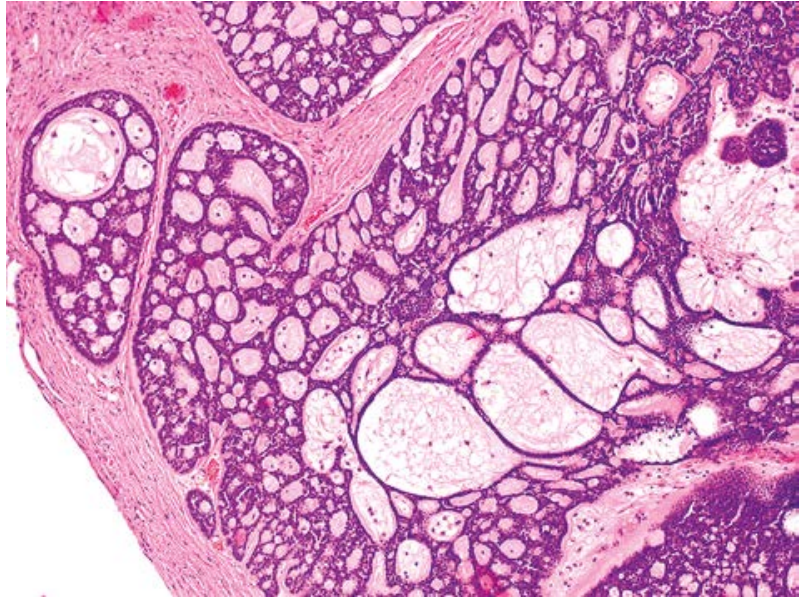

Fig. 4. The same area under higher magnification. HE, magnification 200x

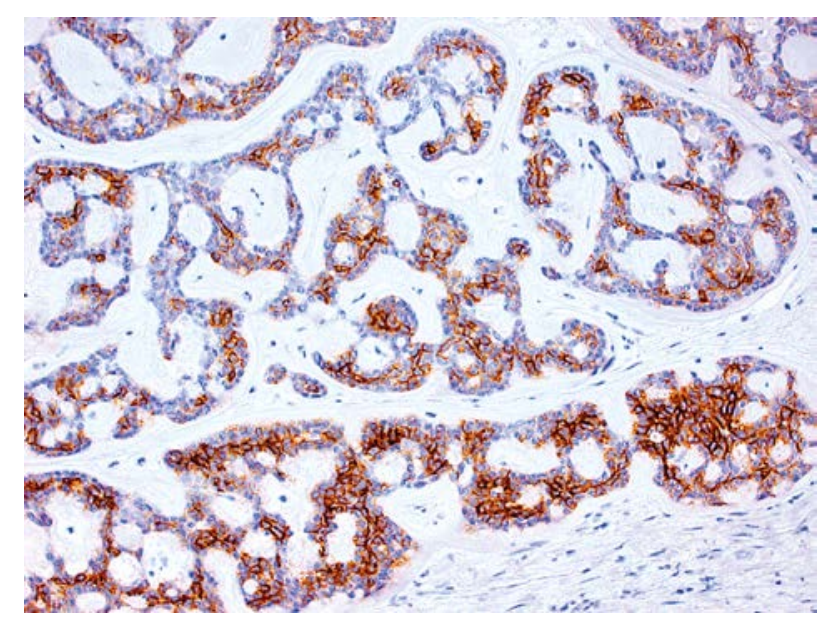

Fig. 6. Immunohistochemistry with Ber_EP4 antibody shows positive reaction in numerous tumour cells. IHC, DAB, magnification 200x

cytoplasm of the inner layer, which forms cellular nests arranged in a mosaic or jigsaw puzzle pattern, separated by relatively thick eosinophilic basal membranes that contain collagen types IV and VII. Tunggal et al. [9] believe their presence to be connected with a laminin-5 defect. There are small spaces in the tumour nests, and the membranes stain PAS-positive. Positive cytokeratin expression confirms the eccrine character of the tumour. The tumour cells show a positive staining with S-100 protein and actin antibodies, emphasizing a possible myoepithelial and glandular eccrine differentiation [10]. Sometimes, small ductal structures are observed in the tumour. Mitoses occur rarely. The characteristic peripheral growth of the tumour, in the form of cleaved nests developing in the hypodermis, does not prejudge its malignant course, nor do cases with increased quantity of mitoses. A malignant course may be suggested by intensified anaplastic histological features and the absence of organoid structures. Cylindroma should be differentiated from basal cell carcinoma and eccrine spiradenoma. Research shows that eccrine spiradenoma, together with cylindroma, form a type of a hybrid displaying similar common features [11]. The diagnosis of spiradenoma is based on the presence of more rounded 
nests in its structure. Basal cell cancer is usually characterised by a connection with the epidermal basal layer, a peripheral palisade-type arrangement of nuclei, cell shrinkage artefacts, necrosis and numerous mitoses. In assessing the primary tumour, differentiation towards cylindroma is not conclusive and displays features common to the entire ontogenetic group of eccrine-apocrine origin. The huge variety of tissue patterns and the clinical course in ovarian teratoma means that, despite attempts at histological classification of lesions into benign or malignant, this group of tumours remains clinically surprising and requires constant monitoring. An accepted mode of therapy in such tumours is surgical resection; additional therapy such as radiation or adjuvant chemotherapy is not used.

The authors wish to thank Mrs Sandra Lindon for linguistic assistance in the preparation of the manuscript.

\section{References}

1. Tavassolli FA, Devilee P. World Health Organization Classification of Tumours. Pathology and Genetics of Tumours of the Brest and Female Genital Organs. IARC Press, Lyon 2003; 169-71.

2. McKenney JK, Soslow RA, Longacre TA. Ovarian mature teratomas with mucinous epithelial neoplasms: morphologic heterogeneity and association with pseudomyxoma peritonei. Am J Surg Pathol 2008; 32: 645-55.

3. Alexander M, Cope N, Renninson J, Hong A, Simpson RH, Hirschowitz L. Relationship between endometriosis, endometrioid adenocarcinoma, gliomatosis peritonei, and carcinoid tumor in a patient with recurrent ovarian teratoma. Int J Gynecol Pathol 2011; 30: 151-7.

4. Targnion A, Broze B, Habonimana E, Jouan H, Frémond B. Gliomatosis peritonei, an unusual abdominal carcinomatosis: report of two cases. Arch Pediatr 2010; 17: 1169-73.

5. Sibio S, Borrini F, Sammartino P, et al. Predominant Brenner tumor combined with struma ovarii containing a papillary microcarcinoma associated with benign peritoneal strumosis: report of a case and histologic features. Endocr Pathol 2010; 21: 199-203.

6. Madan M, Bhagat R, Agarwal AP, Sharma S. Squamous cell carcinoma arising in mature cystic teratoma: a rare case. Indian J Cancer 2010; 47: 346-7.

7. Dos Santos L, Mok E, lasonos A, et al. Squamous cell carcinoma arising in mature cystic teratoma of ovary: A case series and review of the literature. Gynecol Oncol 2007; 105: 321-4.

8. van den Ouweland AM, Elfferich P, Lamping R, van de Graaf R, van Veghel-Plandsoen MM, Franken SM, Houweling AC. Identification of a large rearrangement in CYLD as a cause of familial cylindromatosis. Fam Cancer 2011; 10: 127-32.

9. Tunggal L, Ravaux J, Pesch M, et al. Defective laminin 5 processing in cylindroma cells. Am J Pathol 2002; 160: 459-68.

10. Missall TA, Burkemper NM, Jensen SL, Hurley MY. Immunohistochemical differentiation of four benign eccrine tumors. J Cutan Pathol 2009; 36: 190-6.

11. Rajan N, Burn J, Langtry J, Sieber-Blum M, Lord CJ, Ashworth A. Transition from cylindroma to spiradenoma in CYLD-defective tumours is associated with reduced DKK2 expression. J Pathol 2011; 224: 309-21.

\section{Address for correspondence}

Ewa Zembala-Nożyńska MD, PhD

Wybrzeże Armii Krajowej 15

44-100 Gliwice, Poland

e-mail: jerno@wp.pl

Submitted: 4.07 .2012

Accepted: $\quad 9.04 .2013$ 